\title{
MRI reduces low-risk prostate cancer diagnosis and biopsy
}

An MRI-based biopsy protocol-involving noninvasive screening by $3 \mathrm{~T}$ MRI, followed by selective magnetic resonanceguided biopsy (MRGB) of any identified equivocal or suspicious prostatic lesionshas been shown to reduce the diagnosis of low-risk prostate cancer compared with the current diagnostic standard, transrectal ultrasonography-guided biopsy (TRUSGB).

\section{4 ...almost eliminates the diagnosis of low-risk cancer 77}

In recent years, use of multiparametric (mp) MRI has improved image-based diagnosis of solid organ tumours, but until now it has not been definitively compared with TRUSGB in the same men for detection of prostate cancer. In a study led by Leslie Thompson of The Wesley Hospital in Brisbane, Australia, 223 consecutive biopsy-naive men with elevated PSA and/or abnormal digital rectal examination results were examined using both mpMRI and TRUSGB.
A diagnosis of prostate cancer was made in 126 of the 223 men by TRUSGB, including 47 diagnoses of low-risk cancers. By contrast, 142 men with lesions indicative of intermediaterisk or high-risk disease on mpMRI (scored independently by three readers) underwent MRGB, reducing the need for biopsy by $51 \%$. Prostate cancer was detected in 99 of these men, including only six low-risk cases, a decrease of $89.4 \%$ compared with TRUSGB. The MRI-based protocol "almost eliminates the diagnosis of low-risk cancer," says Thompson, which "relieves a huge psychological, investigative, and treatment burden on men who have been told they have 'insignificant' cancer."

The MRI protocol increased diagnosis of intermediate-risk or high-risk prostate cancer by $17.7 \%$, with 79 diagnoses by TRUSGB and 93 by MRGB. mpMRI with MRGB detected cancers of intermediate or high risk in 29 men that were missed or classified as low risk by TRUSGB. By contrast, 15 cancers designated intermediate or high risk by TRUSGB were either not suggested by mpMRI, or were missed on MRGB. Thompson acknowledges that the MRI methodology is not perfect, but he believes that it can be further improved. "The key to success will be education and quality control," he adds. "Urologists will have to learn about MRI, and learn how to recognize what good image quality is, and insist on this good image quality from their radiologists."

The ability to specifically diagnose high-risk prostate cancer in men with elevated PSA could improve the value of PSA screening by preventing unnecesary biopsies and treatments in men with lowrisk disease. The results suggest that MRI can help to achieve this important aim.

\section{Robert Phillips}

Original article Pokorny, M. R. et al. Prospective study of diagnostic accuracy comparing prostate cancer detection by transrectal ultrasound-guided biopsy versus magnetic resonance (MR) imaging with subsequent MR-guided biopsy in men without previous prostate biopsies. Eur. Urol. doi:10.1016/j.eururo.2014.03.002 